\title{
An incremental modelling approach for the computation of elastic tool effects during metal forming simulations
}

\author{
S. Puchhala, M. Franzke \& G. Hirt \\ Institute of Metal Forming, Aachen, Germany
}

\begin{abstract}
The elastic behaviour of the forming tools has a significant influence on the quality of the end components. For example, in the case of the flat rolling process, the strip quality criteria, i.e., strip flatness and thickness profile are highly influenced by the elastic deformation of the working rolls. The Finite Element (FE) Method is the standard approach for the computation of such forming processes. Considering the elastic effects of the tolls in a single FE model using a traditional approach (standard in the case of commercial FE programs) will lead to large FE models (as very fine descretization of work piece as well as tool at the contact regions is necessary). As a result, the computation requires tremendous amount of time and resources. This paper presents a recently developed concept, which meets the above mentioned demands very efficiently. Within this concept, the computation of the tool elastic effects is separated from the process simulation. The advantages of this concept include, easy handling of the contact situations, reduced FE model size as the tool is modelled as the rigid body and good convergence of the computation. On the other hand, for the validation of the concept, optical measuring techniques were used to validate the numerical simulations. The experimental results of the flat rolling process were compared. To show the portability of the developed concept, qualitative results of the forging process were also given.
\end{abstract}

Keywords: modelling of the flat rolling process, elastic tool behaviour, strip tension modelling, optical measuring techniques. 


\section{Introduction}

In most of the cold forming processes, such as rolling, forging, extrusion and drawing, the end product is aimed to have a better surface quality, closer dimensional tolerances and also improved mechanical properties. Essentially, such processes involve direct contact of the work piece with the tool. The tool force must exceed the elastic limit of the work piece to cause the plastic flow. Due to the required high forces, tools may experience deformations which will directly influence the undesired changes in the end profile of the product.

In the final stage, production processes such as the rolling process, there is no other forming step which has an impact on the thickness profile in a corrective manner. On the other hand, the thickness profile has a substantial influence on the following forming process steps, i.e., the deep drawing process. The thickness profile along the strip width depends on the stress distribution along the roll gap. In other words, due to the elastic work roll deformation, an inhomogeneous contour of the roll gap will be developed. As a result, depending on the roll deformation, crowns will originate within the strip (Figure 1). These tool deformations may also occur in the other forming processes and will significantly affect the quality of the end product.
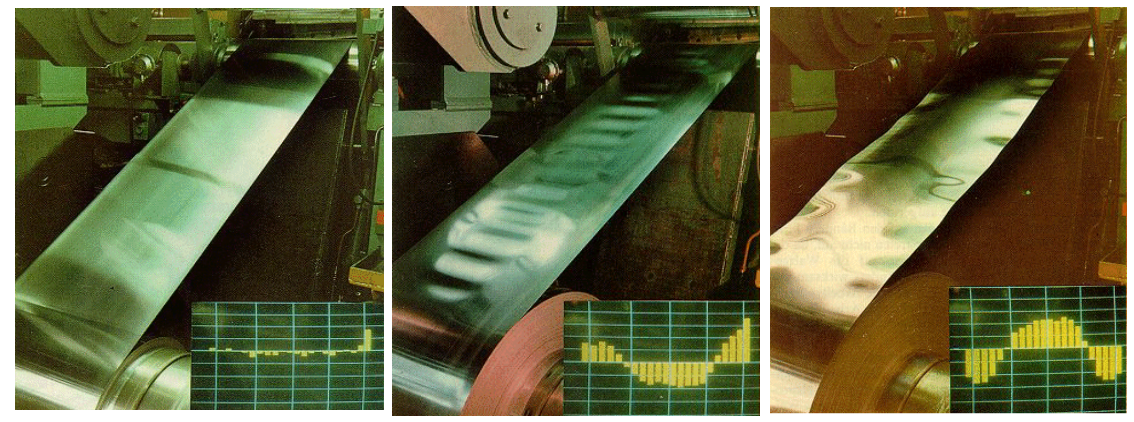

Figure 1: Quality problems during steel strip production.

Modelling such processes in a single numerical model considering all the above mentioned effects, will not only complicate the computation but also affects the convergence criteria. This situation is even more complicated when contact between elastic tools has to be considered. For example, in the case of multi stage rolling mills (quarto configuration), the contact between working rolls and backup rolls. On the other hand, due to highly localized deformations within the work piece, utilization of rigid tools within the uniform profiles (throughout the process) will lead to unreliable predictions.

To ensure the practice, relevant numerical simulation results, prediction of the interaction effects between processes and elastic tools is highly essential. This paper presents a novel approach to accurately model the above mentioned interaction effects. As an example application, the flat rolling process and closed die forging process were modelled. To validate the numerical simulations, 
rolling experiments were conducted and with the help of optical measuring techniques, the strip surface profile (using 3D digitizing system ATOS [1]) and the deformation of the whole mill (using coordinate measuring technique TRITOP [1]) were measured. To demonstrate the portability of the developed concept, the forging process was modelled and qualitative simulation results will also be presented. The experimental setup and experimental techniques were also discussed.

\section{Concept and modelling}

\subsection{Concept of the finite element method-finite element method coupling model}

The computation of the forming process simulations considering the elastic tool effects is separated into two different simulation models. In this context, the forming process simulation consists of the work piece (modelled using elasticplastic material description) and the tools were modelled as rigid bodies. On the other hand, a different simulation model for tools is modelled considering the linear elastic material description. These two separate simulation models are coupled using a module called DOELTOSIM (DO ELastic TOol SIMulation). This module automatically couples both simulation models using a mapping table. This kind of incremental modelling is schematically shown in Figure 2.

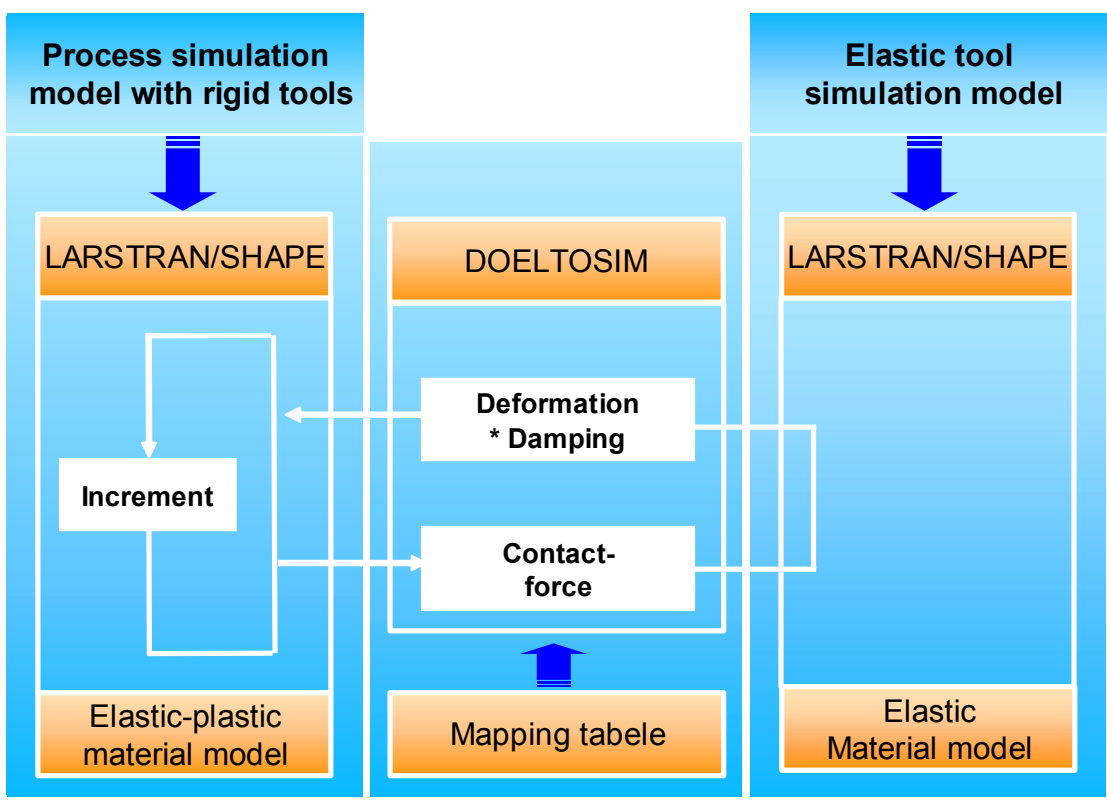

Figure 2: Concept of the finite element method-finite element method coupling model. 
The incremental data exchange between two simulation models is carried out by the DOELTOSIM module. This module uses a mapping table to update the geometry information (node and element connectivity) in both simulation models. For the communication and compatibility between two models, an equivalent mesh will be used in both simulation models for the tools and this information is maintained in the mapping table. At predefined time steps, the normal contact forces (at the contact nodes) were extracted from the process simulation model and are automatically applied onto the corresponding contact nodes in the elastic simulation model using the mapping table. For the contact formulation, LARSTRAN/SHAPE [2] uses classical penalty contact description according to the master-slave approach. The contact definition is required only in the process simulation model, where the tools are considered as master and the work piece s modelled as slave body.

As shown in Figure 2, the coupled simulation must be started with the process simulation. The activation of the elastic tool simulation is controlled from the process simulation using the time steps. At predefined time step, a new elastic simulation will be carried out and the deformed tool geometry will be uploaded into the process simulation. A damping factor was introduced to avoid the unwanted oscillations.

\subsection{Modelling of the forming process}

As described in the previous section, the process simulation is separated into two different simulations. Within the process simulation model, the tools are modelled as rigid bodies and the work piece is modelled as deformable model with the elastic-plastic material description. On the other hand, pure linear elastic material description was used to model the tools in a separate simulation model. These two simulation models communicate using mapping table. This mapping table consists of the surface element data and node information of rigid body (of process simulation model) and elastic body (of elastic simulation model). The surface mesh geometry of both simulation models should be coincide each other to have one-to-one mapping. The rigid tool geometry was generated from the elastic tool sing a function implemented into the pre- and postprocessor called PEP [3]. This surface geometry in both simulation models must not be changed throughout the simulation. A typical finite element (FE)-mesh used to model flat rolling process tools (working rolls) is shown in Figure 3. Only the surface mesh (used as rigid body) is being used in the process simulation model and the equivalent volume mesh is being used in the corresponding elastic tool simulation model.

The steady state criterion is most important in this kind of coupled simulation models. This directly depends on the frequency of the activation of the elastic tool simulations. In other sense, after each elastic tool simulation, the rigid tool geometry within the process simulation model will be updated which means a new roll gap will e generated. To attain the steady state, the strip must achieve the new roll gap. Once the strip has achieved the new roll gap, the simulation will be steady and only after this condition, a new elastic tool simulation must be activated. 


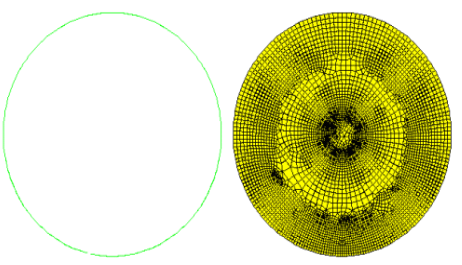

(a) With 4838 elements and 4393 nodes.
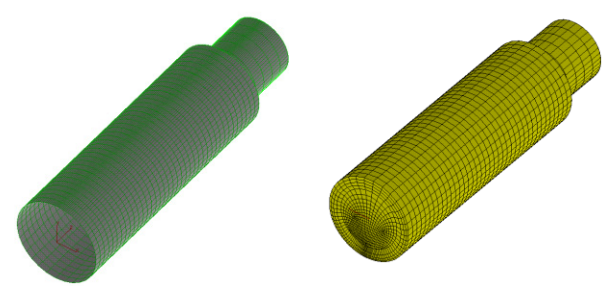

(b) With 16,140 elements and 17,760 nodes.

Figure 3: $\quad$ FE mesh for the rolling process tools (a) 2D model with rigid tool (left) and corresponding elastic tool (right) (b) 3D model.

\section{Numerical simulation models}

\subsection{Flat rolling process simulation}

As an example application of the concept described in the previous section, a cold rolling process was investigated using 2D and 3D FE models. For a profound understanding of the plastic deformation, an implicit solver was used. The work piece, i.e., strip, was modelled using a visco-plastic material description with 4-node quadrilateral elements for 2D models and 8-node hexahedral elements for $3 \mathrm{D}$ models. According to the process, a mid plane symmetry property was used. For a clear understanding of the interaction behaviour, extremely fine mesh was used at the contact zones for both work piece and tools. The model geometrical dimensions and FE mesh information is given in table 1. Within the simulation models, it was necessary to apply an initial motion to the strip to move it into the roll gap. Once the strip was in the roll gap, the external motion was taken out.

A strip of initial thickness of $1.67 \mathrm{~mm}$ was rolled to 4 different thickness reductions $(1.2 \%, 11.3 \%, 27 \%$ and $28.3 \%)$ using elastic rolls in a duo configuration. The initial radius of the work roll was $299.2 \mathrm{~mm}$ and was rotating with a velocity of $1 \mathrm{rad} / \mathrm{sec}$. The initial strip velocity of $1.07 \mathrm{~mm} / \mathrm{sec}$ was applied using an external rigid tool. The velocity of the strip coincides with the initial roll velocity which ensures minimum distortion of the strip elements at the initial stage. A tensile test was carried out to measure the yield stress curve for the strip material (ZStE340). The maximum measured true strain is about 0.15 , which represents a $11.3 \%$ thickness reduction. Further true strain values were approximated with the regression equation (Equation (1)) according to LudwikHolomon [5].

$$
k_{f}=a+b \cdot \varphi^{n} \text {, with coefficients } \mathrm{a}=210 \mathrm{MPa}, \mathrm{b}=435 \mathrm{MPa} \text { and } \mathrm{n}=0.26
$$


Forward and backward strip forces of 20 and $30 \mathrm{kN}$ were applied respectively on the strip cross-section and kept constant during the whole simulation. Young's modulus of 185E3 N/mm ${ }^{2}$ was chosen for the strip and $225 \mathrm{E} 3 \mathrm{~N} / \mathrm{mm}^{2}$ was chosen for the working roll. Poisson's ratio for both strip and roll was chosen as 0 . The friction coefficient between strip and roll was modelled using Coulomb friction law with coefficient $\mu=0.3$.

\section{Experimental setup and measurements}

The cold rolling experiments were carried out on a rolling mill installed at the Institute of Metal Forming. A photograph of the mill is shown in Figure 4. The rolling mill can be used as duo (a pair of working rolls) or as a quarto configuration (a pair of working rolls and a pair of back up rolls). The diameter of the working rolls is $135.3 \mathrm{~mm}$ and the supporting rolls is $299.2 \mathrm{~mm}$. Single pass experiments were conducted in duo configuration using the strip material ZStE340 with the initial thickness of $1.67 \mathrm{~mm}$.

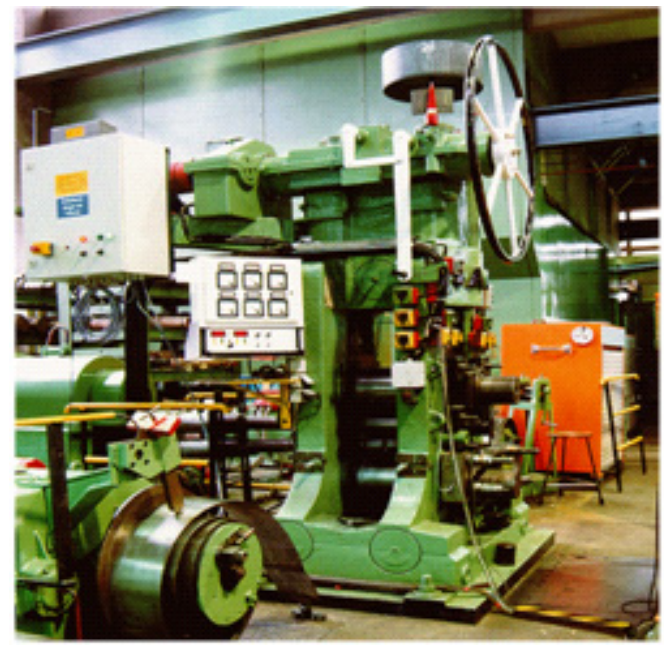

Figure 4: $\quad$ Rolling mill installed at IBF.

The strip plastic strain distribution is measured using the optical strain measuring system, ARGUS. Before rolling, a regular dot pattern was glued onto the strip. The dot pattern distorts with the strip plastic deformation during rolling. The deformed strip is then recorded from different angles using highresolution $\mathrm{CCD}$ cameras. By using the photo grammetric evolution procedures, the position of all 3D points (strain distribution) is automatically captured using the ARGUS software program.

The surface profile of the rolled strip was measured using the $3 \mathrm{D}$ digitalizing system, ATOS. This is optical measuring equipment based on the principle of triangulation. A sensor is positioned in front of the rolled strip. Fringe patterns 
are then projected onto the strip surface (using white light projection) and the profile is recorded by two cameras. Using the software provided by the equipment, high-precision $3 \mathrm{D}$ coordinate points of the surface profile were captured.

Further, by utilising the optical coordinate measuring techniques, TRITOP, the deformation of the whole rolling mill was measured. Images were taken before, during and after the rolling process from different view angles using a digital photo grammetric camera. From these images, TRITOP's software automatically calculates the $3 \mathrm{D}$ coordinate of the measuring points. The difference of the coordinate points before and after rolling process is then measured.

\section{Numerical simulation and experimental results}

The rolling process under consideration was simulated by utilizing the PEP and LARSTRAN/SHAPE (an implicit solver). To obtain reliable steady state results, at least 5 to 10 deformed contact lengths were simulated. For all 3D test cases, rolling of the length of $100 \mathrm{~mm}$ takes approximately 2.4 seconds real process time. This required about 2000 time increments with a stable increment order of 0.001. This led the simulation computation time of 60 hours and 45 minutes (including the Input/Output) for each coupled cycle the elastic simulation took 6 minutes and 30 seconds to update the elastic effects into the process simulation model. At the same time, a 3D rolling test simulation using elastic tools in a single FE model (using identical FE models used in out tests) was conducted using ABAQUS/STANDARD, and required 225 hours which corresponds to about 4 times slower than the model presented here.

The computed and measured rolling forces are compared and are shown in Figure 5 . Up to the $11 \%$ thickness reduction, both the rigid and deformable tool

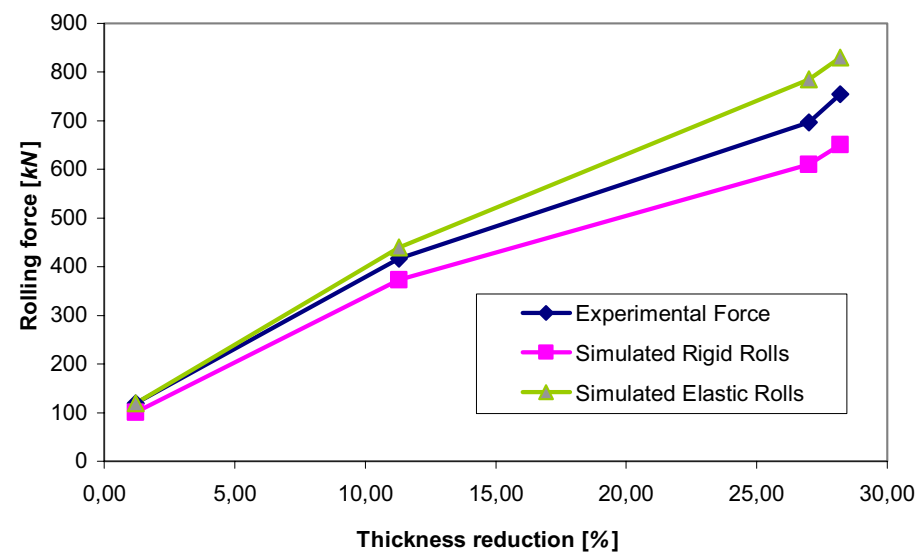

Figure 5: $\quad$ Comparison of measured and computed rolling force. 
504 High Performance Structures and Materials V

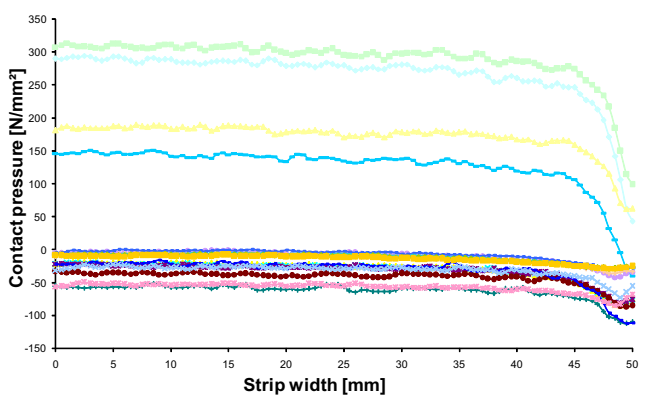

(a)

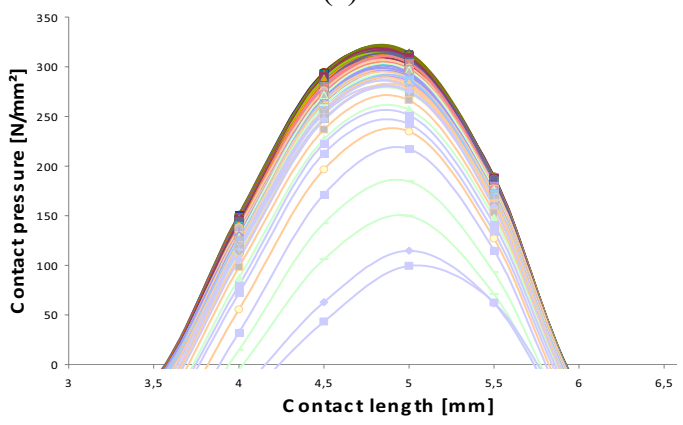

(b)

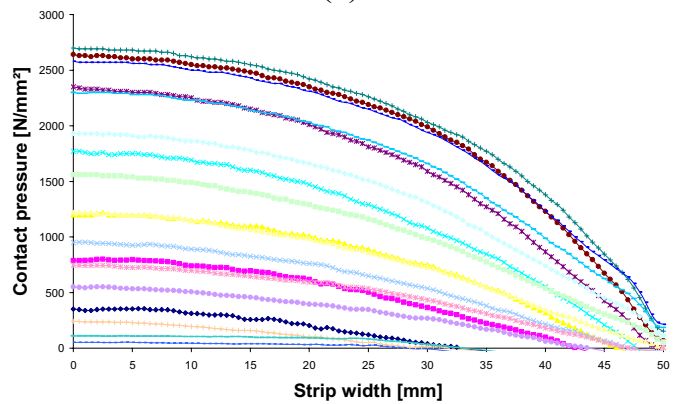

(c)

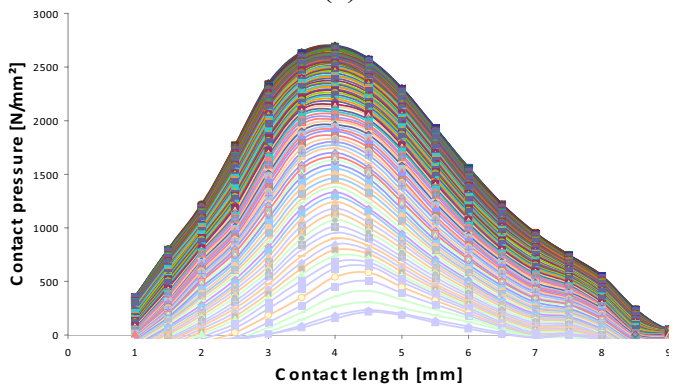

(d)

Figure 6: Contact pressure distribution along the strip width and contact length direction. 


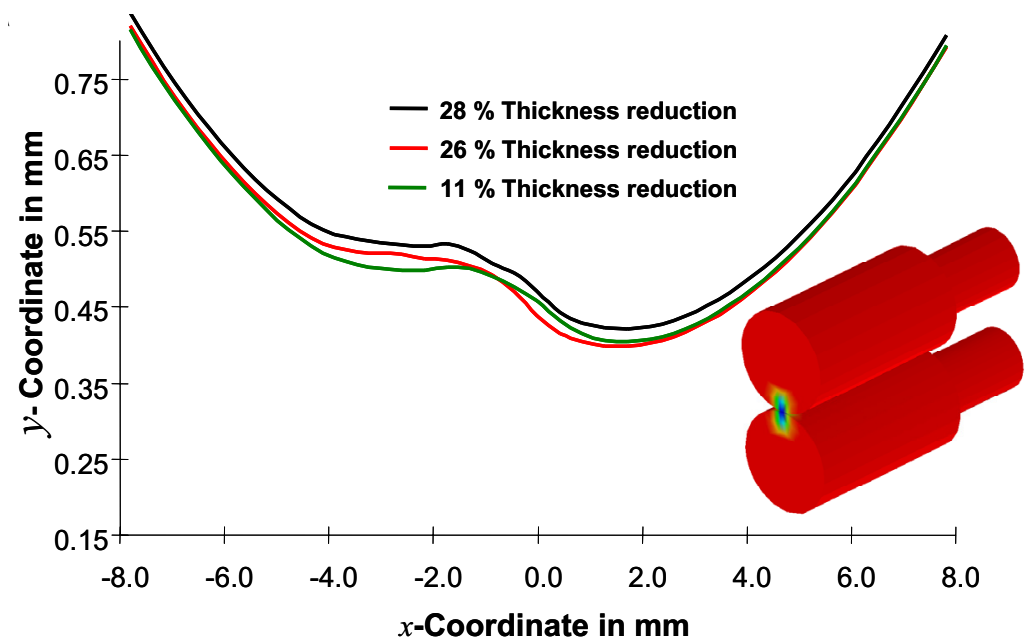

(a)

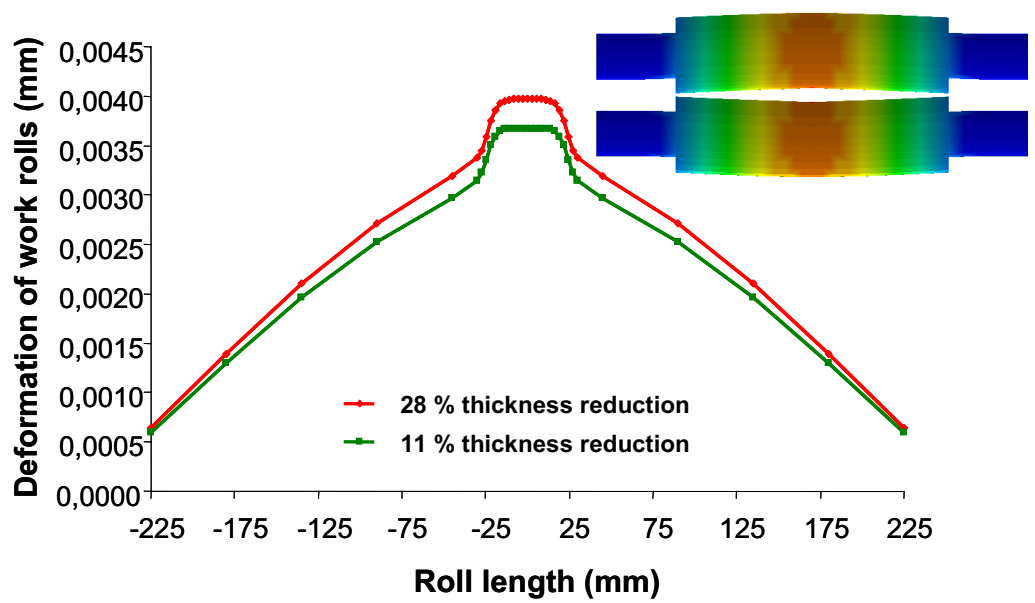

(b)

Figure 7: Work roll deformation along the cross-section and the roll length.

simulation results match very well. However, after a $20 \%$ thickness reduction, the simulation results have a tendency of deviation. The elastic tool model overestimated the force and at the same time the rigid tool model delivered relatively low force values. Due to high work roll deformation and high friction coefficient $(\mu=0.3)$ the elastic rolls model overestimated the rolling force values. With the variation of friction coefficient this situation may be improved.

Due to the work roll deformation, the contact pressure distribution changes over strip width and also in the contact length direction. Figure 6 presents the 
steady state contact pressure (linear row of nodes selected over the strip width) using deformable rolls. In the same way, the contact pressure in the contact length direction is shown in Figure 6(b).

As expected, due to the non-uniform force distribution during large thickness reduction cases, the contact pressure distribution drastically changes along the strip width. At the same time, depending on the thickness reduction $(1.2 \%$ and $28.3 \%$ ), the contact length also changes (Figure 6(d)). On the other hand, the work roll deformation along the roll cross-section is given in Figure 7.

To show the portability of the concept developed, a forging process was also modelled and qualitative results are shown in Figure 8. As shown in Figure 8(a), the lower tool was modelled as elastic body and the corresponding deformation distribution is shown in Figure 8(b).

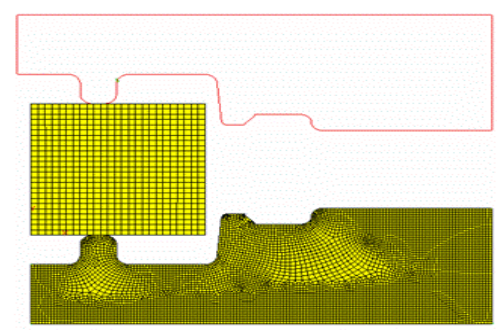

(a)

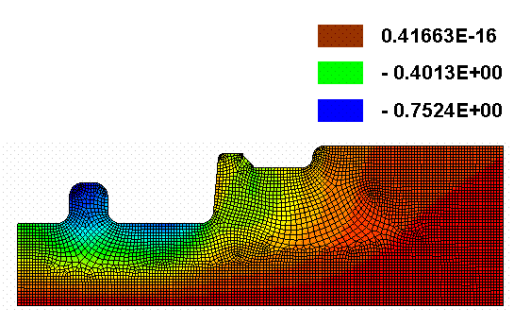

(b)

Figure 8: $\quad$ FE model and deformation distribution of the forging process.

\section{Outlook and conclusion}

Computation of deformation effects during metal forming processes using a recently developed incremental modelling approach is clearly described with example applications. This paper presents the concept of separating the process simulation with the elastic tool simulation, which led to simple handling and reliable computation time. It is shown in the modelling and results sections that the proposed modelling approach is highly general and can be applied to various forming processes. As this work is under development, a number of computations and experiments have to be taken into account. The heat transfer effects of the tools during hot forming processes have to be implemented.

\section{Acknowledgements}

The authors would like to thank "Deutsche Forschungsgemeinschaft" (DFG) for their continuous support through the project "Interaction effects between process and structures" - SPP1180. 


\section{References}

[1] K. Glanulis, Optical measuring techniques in sheet metal processes GOM Gesselschaft fuer Optische Messtechnik GmbH.

[2] A. Kurz, 2004, LARSTRAN/SHAPE user's manual, LASSO engineer gesselschaft

[3] M. Franzke, 2007, PEP User's manual, ACUT GmbH, Aachen

[4] S. Puchhala, M. Franzke, H. Dackweiler: Modelling interaction effects during flat rolling process, NUMIFORM 2007, Porto

[5] P. Ludwik, 1909, Elemente der Technologischen Mechanik, SpringerVerlag, Berlin 